\title{
Oscillating chiral currents in nanotubes: a route to nanoscale magnetic test tubes.
}

\author{
C. J. Lambert and S. W. D. Bailey \\ Department of Physics, Lancaster University, Lancaster, LA1 4YB, U. K. \\ J. Cserti \\ Department of Physics of Complex Systems, Eötvös University, H-1117 Budapest, Hungary
}

(Dated: April 22, 2019)

\begin{abstract}
With a view to optimising the design of carbon-nanotube (CNT) windmills and to maximising the internal magnetic field generated by chiral currents, we present analytical results for the group velocity components of an electron flux through chiral carbon nanotubes. Chiral currents are shown to exhibit a rich behaviour and can even change sign and oscillate as the energy of the electrons is increased. We find that the transverse velocity and associated angular momentum of electrons is a maximum for non-metallic CNTs with a chiral angle of $18^{\circ}$. Such CNTs are therefore the optimal choice for CNT windmills and also generate the largest internal magnetic field for a given longitudinal current. For a longitudinal current of order $10^{-4}$ amps, this field can be of order $10^{-1}$ Teslas, which is sufficient to produce interesting spintronic effects and a significant contribution to the self inductance.
\end{abstract}

PACS numbers: 73.63.-b,68.65.-k,71.15.Ap

Chiral nanotubes and nanowires are of interest for a range of properties associated with external magnetic fields 1], their potential for creating nanoscale inductors [2] and their suggested role as building blocks in chiral nanomotors [3]. Examples studied to date include chiral carbon and $\mathrm{BC} 2 \mathrm{~N}$ nanotubes [4, 5, 6], BN nanotubes [7], Fe-filled carbon nanotubes (CNTs) [8] and chiral singlewall gold nanotubes [9].

Most recently, interest in chiral currents has been rekindled by their potential to drive CNT nanometrescale motors 10, 11, 12. Such motors benefit from low inter-wall friction 13 and a high tensile strength 14], which allow one to engineer complex structures 15, 16, 17], including nanoscale bearings [10, 18], rotors 17, 19], oscillators 20, 21, 22, 23], switches 24] and telescopes [25]. The helical arrangement of the atoms in chiral CNTs can be exploited 26, 27] to produce a Brownian ratchet effect 3], rotational and translational motion driven by thermal gradients [28, 29] and motion [30] induced by circularly polarized light. Recently a new drive mechanism for CNT windmills was proposed [31], based upon the torque generated by a flux of electrons passing through a chiral CNT. It was shown that under appropriate conditions, the dominant contribution to this torque is proportional to the flux of angular momentum carried by electrons moving in the corresponding infinite chiral CNT. This provides a useful guide for the design of CNT windmills, since a calculation of the angular momentum and associated chiral currents carried by electrons in infinite chiral nanotubes does not require the solution of a scattering problem.

The fact that large chiral currents occur in CNTs is at first sight surprising, since early first-principles studies [2, 4] suggested that chiral CNTs do not carry a significant chiral current. In this paper we study the energy and voltage dependence of chiral currents in CNTs and show that the small chiral current found in [4] is a consequence of the metallic nature of the CNT studied. In contrast for non-metallic CNTs, we predict much larger chiral currents. We find that the energy dependence of chiral currents is surprisingly rich. Indeed the transverse current components can even change sign and oscillate as the energy of the electrons is increased. We demonstrate that the presence of large chiral currents produces significant magnetic fields of order 0.1 Teslas within the volume of a CNT, thereby providing a novel magnetic test tube, which could be used to manipulate the magnetic moments of encapsulated magnetic molecules or particles. This internal field produces a significant contribution to the self inductance of the CNT, which must be added to the more usual contribution associated with the external magnetic field 32].

To define the velocity components of electrons in chiral CNTs, we follow the notation of [33], which introduces the lattice vectors of the corresponding infinite $2 \mathrm{D}$ graphene sheet, defined by $\mathbf{a}_{\mathbf{1}}=\left(\frac{\sqrt{3}}{2}, \frac{1}{2}\right) \mathrm{a}$ and $\mathbf{a}_{2}=\left(\frac{\sqrt{3}}{2},-\frac{1}{2}\right)$ a, where $a=\sqrt{3} a_{c-c}$ and $a_{c-c}=1.44 \AA$ is the carbon-carbon bond length. An (n, m) CNT, where $(0 \leq \mathrm{m} \leq \mathrm{n})$ are integers, is then defined by a transverse chiral vector $\mathbf{C h}=n \mathbf{a}_{\mathbf{1}}+\mathbf{m a}_{\mathbf{2}}$, which wraps around the CNT circumference and a longitudinal translation vector $\mathbf{T}$. We are interested in resolving electron velocities along axes $\mathrm{x}$ and $\mathrm{y}$, which are parallel to the unit vectors $\hat{\mathbf{C h}}$ and $\hat{\mathbf{T}}$ respectively. The velocity components are given by $\hbar \mathrm{v}_{\mathrm{x}}=\partial \mathrm{E}(\mathbf{k}) / \partial \mathrm{k}_{\mathrm{x}}$ and $\hbar \mathrm{v}_{\mathrm{y}}=\partial \mathrm{E}(\mathbf{k}) / \partial \mathrm{k}_{\mathrm{y}}$, where $\mathrm{k}_{\mathrm{x}}=\mathbf{k} \cdot \hat{\mathbf{C h}}, \mathrm{k}_{\mathrm{y}}=\mathbf{k} \cdot \hat{\mathbf{T}}$ and $\mathrm{E}(\mathbf{k})$ is the energy dispersion relation. In the simplest Slater-Koster scheme, $\mathrm{E}(\mathbf{k})$ takes the form

$$
\mathrm{E}(\mathbf{k})=\gamma\left|1+\exp \left(-\mathrm{ik} \cdot \mathbf{a}_{\mathbf{1}}\right)+\exp \left(-\mathrm{ik} \cdot \mathbf{a}_{2}\right)\right|,
$$

where $\gamma$ is the hopping integral. 
Each mini band possesses a continuous longitudinal wave vector $\mathrm{k}_{\mathrm{y}}$ and is labelled by a quantised value of $\mathrm{k}_{\mathrm{x}}$, given by

$$
\mathrm{k}_{\mathrm{x}}^{\mathrm{q}}=\frac{2 \pi \mathrm{q}}{|\mathbf{C h}|}, \quad\left(\mathrm{q}=1, \ldots \mathrm{N}_{\text {hex }}\right)
$$

where $\mathrm{N}_{\text {hex }}$ is the number of hexagons in a CNT unit cell. For a given choice of $\mathrm{E}$ and $\mathrm{q}$, equation (1) can be solved to yield two values of $\mathrm{k}_{\mathrm{y}}$. One of these values, which we denote $\mathrm{k}_{\mathrm{y}}^{+}(\mathrm{q}, \mathrm{E})$, corresponds to a positive longitudinal velocity $\mathrm{v}_{\mathrm{y}}\left(\mathrm{q}, \mathrm{k}_{\mathrm{y}}^{+}(\mathrm{q}, \mathrm{E})\right.$. The other value, which we denote $\mathrm{k}_{\mathrm{y}}^{-}(\mathrm{q}, \mathrm{E})$, corresponds to a negative longitudinal velocity $\mathrm{v}_{\mathrm{y}}\left(\mathrm{q}, \mathrm{k}_{\mathrm{y}}^{-}(\mathrm{q}, \mathrm{E})\right.$. In what follows, we refer to these electrons as "right-moving" and "left-moving" respectively. We are interested in the transverse velocities of right-moving electrons, which we denote by $\mathrm{v}_{\mathrm{x}}\left(\mathrm{q}, \mathrm{k}_{\mathrm{y}}^{+}(\mathrm{q}, \mathrm{E})\right)$. Our aim is to compute the total transverse velocity $v_{x}^{(n, m)}(E)$ of all right-moving electrons of energy E, which in units of the Fermi velocity $v_{F}$ is

$$
\mathrm{v}_{\mathrm{x}}^{(\mathrm{n}, \mathrm{m})}(\mathrm{E})=\sum_{\mathrm{q}} \mathrm{v}_{\mathrm{x}}\left(\mathrm{q}, \mathrm{k}_{\mathrm{y}}^{+}(\mathrm{q}, \mathrm{E})\right) / \mathrm{v}_{\mathrm{F}}
$$

where the sum is over all mini bands with real longitudinal wave vectors of energy E.

At low-enough energies, the wave vectors of mini band $\mathrm{q}$ [namely $\left(\mathrm{k}_{\mathrm{x}}^{\mathrm{q}}, \mathrm{k}_{\mathrm{y}}^{+}(\mathrm{q}, \mathrm{E})\right)$ and $\left.\left(\mathrm{k}_{\mathrm{x}}^{\mathrm{q}}, \mathrm{k}_{\mathrm{y}}^{-}(\mathrm{q}, \mathrm{E})\right)\right]$ can be chosen to be close to the $K$ point $K$. Since $E(\mathbf{k})$ is an even function of $\mathbf{k}$, there will be another pair of wave vectors in the vicinity of the second $K$ point $-\mathbf{K}$, given by $\left(\mathrm{k}_{\mathrm{x}}^{\mathrm{q}^{\prime}}, \mathrm{k}_{\mathrm{y}}^{-}\left(\mathrm{q}^{\prime}, \mathrm{E}\right)\right)=\left(-\mathrm{k}_{\mathrm{x}}^{\mathrm{q}},-\mathrm{k}_{\mathrm{y}}^{+}(\mathrm{q}, \mathrm{E})\right)$ and $\left(\mathrm{k}_{\mathrm{x}}^{\mathrm{q}^{\prime}}, \mathrm{k}_{\mathrm{y}}^{+}\left(\mathrm{q}^{\prime}, \mathrm{E}\right)\right)=-\left(\mathrm{k}_{\mathrm{x}}^{\mathrm{q}}, \mathrm{k}_{\mathrm{y}}^{-}(\mathrm{q}, \mathrm{E})\right)$, which possess negative and positive longitudinal group velocities respectively.

The contribution to the sum in equation (3) from these two mini bands is

$$
\begin{array}{r}
\mathrm{v}_{\mathrm{x}}(\mathrm{q}, \mathrm{E})=\mathrm{v}_{\mathrm{x}}\left(\mathrm{q}, \mathrm{k}_{\mathrm{y}}^{+}(\mathrm{q}, \mathrm{E})\right)+\mathrm{v}_{\mathrm{x}}\left(\mathrm{q}^{\prime}, \mathrm{k}_{\mathrm{y}}^{+}\left(\mathrm{q}^{\prime}, \mathrm{E}\right)\right) \\
=\mathrm{v}_{\mathrm{x}}\left(\mathrm{q}, \mathrm{k}_{\mathrm{y}}^{+}(\mathrm{q}, \mathrm{E})\right)+\mathrm{v}_{\mathrm{x}}\left(-\mathrm{q},-\mathrm{k}_{\mathrm{y}}^{-}(\mathrm{q}, \mathrm{E})\right) \\
=\mathrm{v}_{\mathrm{x}}\left(\mathrm{q}, \mathrm{k}_{\mathrm{y}}^{+}(\mathrm{q}, \mathrm{E})\right)-\mathrm{v}_{\mathrm{x}}\left(\mathrm{q}, \mathrm{k}_{\mathrm{y}}^{-}(\mathrm{q}, \mathrm{E})\right)
\end{array}
$$

The last line in this expression is useful, because it allows us to focus on the contributions from a single $\mathrm{K}$ point only. It also demonstrates that a non-zero transverse velocity arises from trigonal warping, since for a perfect Dirac cone, the right hand side of equation (4) would vanish. This suggests that at low energies, an analytical expression for $\mathrm{v}_{\mathrm{x}}(\mathrm{q}, \mathrm{E})$, can be obtained by writing the electron wave vector in the form $\mathbf{k}=\mathbf{K}+\boldsymbol{\eta}$, where $\mathbf{K}=(0,1) 4 \pi /(3 \mathrm{a})$ is a vector pointing from the origin to a K-point and Taylor expanding $\mathrm{E}(\mathbf{k})$ as a power series in $\eta_{\mathrm{x}}$ and $\eta_{\mathrm{y}}$, where $\eta_{\mathrm{x}}=\boldsymbol{\eta} . \hat{\mathbf{C h}}$ and $\eta_{\mathrm{y}}=\boldsymbol{\eta} . \hat{\mathbf{T}}$. This expansion is of the form

$$
\mathrm{E}^{2}=\sum_{\mathrm{i}, \mathrm{j}=0}^{\infty} \mathrm{c}_{\mathrm{ij}} \eta_{\mathrm{x}}^{\mathrm{i}} \eta_{\mathrm{y}}^{\mathrm{j}}
$$

where the coefficients $\mathrm{c}_{\mathrm{ij}}$ satisfy $\mathrm{c}_{00}=\mathrm{c}_{10}=\mathrm{c}_{01}=$ $\mathrm{c}_{11}=0$ and $\mathrm{c}_{02}=\mathrm{c}_{20}=3 \gamma^{2} /\left(4 \mathrm{a}^{2}\right), \mathrm{c}_{21}=$ $-\mathrm{c}_{03} / 3=27 \gamma^{2} \mathrm{a}^{3} \mathrm{mn}(\mathrm{m}+\mathrm{n}) / 16\left(\mathrm{n}^{2}+\mathrm{m}^{2}+\mathrm{nm}\right)^{3 / 2}, \mathrm{c}_{12}=$ $3 \sqrt{3} \gamma^{2} a^{3}(m-n)(2 n+m)(2 m+n) / 16\left(n^{2}+m^{2}+n m\right)^{3 / 2}$. Differentiating this with respect to $\eta_{\mathrm{x}}$ and writing $\left(\mathrm{k}_{\mathrm{x}}^{\mathrm{q}}, \mathrm{k}_{\mathrm{y}}^{ \pm}(\mathrm{q}, \mathrm{E})\right)=\mathbf{K}+\left(\eta_{\mathrm{x}}, \eta_{\mathrm{y}}^{ \pm}\right)$, yields to order $\left.\left[\eta_{\mathrm{y}}^{ \pm}\right]^{2}\right]\left[\eta_{\mathrm{x}}\right]$,

$$
\begin{aligned}
& 2 \operatorname{Ev}_{\mathrm{x}}(\mathrm{q}, \mathrm{E})=\mathrm{c}_{12}\left[\left(\eta_{\mathrm{y}}^{+}\right)^{2}-\left(\eta_{\mathrm{y}}^{-}\right)^{2}\right] \\
& \quad+\eta_{x}\left\{2 c_{21}\left[\eta_{y}^{+}-\eta_{y}^{-}\right]+2 c_{22}\left[\left(\eta_{y}^{+}\right)^{2}-\left(\eta_{y}^{-}\right)^{2}\right]\right\}
\end{aligned}
$$

To compute $\eta_{\mathrm{y}}^{ \pm}$for fixed $\eta_{\mathrm{x}}$ and $\mathrm{E}$, we consider two cases: The first case arises when $\eta_{\mathrm{x}} \neq 0$, in which case one obtains

$$
\mathrm{v}_{\mathrm{x}}(\mathrm{q}, \mathrm{E}) / \mathrm{v}_{\mathrm{F}}=\frac{2 \eta_{\mathrm{x}} \mathrm{c}_{21}}{\mathrm{E}} \sqrt{\mathrm{E}^{2} / \mathrm{c}_{02}-\eta_{\mathrm{x}}^{2}}
$$

The second case corresponds to $\eta_{\mathrm{x}}=0$. In this case, equation (5) yields to lowest order, $\mathrm{E}^{2}=\mathrm{c}_{02} \eta_{\mathrm{y}}^{2}+\mathrm{c}_{03} \eta_{\mathrm{y}}^{3}$, which after solving by iteration and combining with equation (6) yields

$$
\mathrm{v}_{\mathrm{x}}(\mathrm{q}, \mathrm{E}) / \mathrm{v}_{\mathrm{F}}=-\mathrm{c}_{03} \mathrm{c}_{12} \mathrm{E}^{2} / \mathrm{c}_{02}^{5 / 2}
$$

where $\mathrm{v}_{\mathrm{F}}=\mathrm{c}_{02}^{1 / 2} / \hbar=\sqrt{3} \gamma / 2 \mathrm{a} \hbar$ is the Fermi velocity.

Since $\mathrm{k}_{\mathrm{x}}=2 \pi \mathrm{q} /|\mathbf{C h}|$ and $\mathrm{K}_{\mathrm{x}}=\mathbf{K} \cdot \hat{\mathbf{C h}}=2 \pi(\mathrm{n}-$ $\mathrm{m}) / 3|\mathbf{C h}|$, the value of $\eta_{\mathrm{x}}$ for the lowest-energy mini band is $\eta_{1}=2 \pi(-\mathrm{X} / 3) /|\mathbf{C h}|$, where $\mathrm{X}=1$ for $\mathrm{n}=\mathrm{m}+1$, $\mathrm{n}=\mathrm{m}+4$, etc, whereas $\mathrm{X}=-1$ for $\mathrm{n}=\mathrm{m}-1, \mathrm{n}=\mathrm{m}+2$, $\mathrm{n}=\mathrm{m}+5$, etc and $\mathrm{X}=0$ for $\mathrm{n}=\mathrm{m}, \mathrm{n}=\mathrm{m} \pm 3$, etc. Values of $\eta_{\mathrm{x}}$ for higher-energy mini bands are obtained from $\eta_{1}$ by adding or subtracting integer multiples of $2 \pi / \mid \mathbf{C h}$. For non-metallic CNTs, where $\mathrm{X}= \pm 1$, the value of $\eta_{\mathrm{x}}$ for the second mini band is $\eta_{2}=2 \pi(2 \mathrm{X} / 3) /|\mathbf{C h}|$, whereas for metallic CNTs, $\eta_{2}= \pm 2 \pi /|\mathbf{C h}|$.

For $\eta_{\mathrm{j}} \neq 0$ equation (7) yields for the dimensionless transverse velocity associated with channel $\eta_{\mathrm{j}}$ of a $(\mathrm{n}, \mathrm{m})$ CNT,

$$
\mathrm{v}_{\mathrm{x}}^{(\mathrm{n}, \mathrm{m})}\left(\eta_{\mathrm{j}}, \mathrm{E}\right)=\mathrm{v}_{\mathrm{x}}(\mathrm{q}, \mathrm{E}) / \mathrm{v}_{\mathrm{F}}=\mathrm{v}_{\mathrm{j}} \epsilon_{\mathrm{j}}^{3 / 2}\left(\epsilon-\epsilon_{\mathrm{j}}\right)^{1 / 2} / \epsilon,
$$

where

$$
\mathrm{v}_{\mathrm{j}}=3 \sqrt{6} \mathrm{mn}(\mathrm{n}+\mathrm{m}) /\left(\mathrm{n}^{2}+\mathrm{m}^{2}+\mathrm{mn}\right)^{3 / 2}\left[\operatorname{sign} \text { of } \eta_{\mathrm{j}}\right] .
$$

In this expression, $\epsilon=\mathrm{E} / \gamma$ and $\epsilon_{j}$ is the energy minimum of the jth mini band (in units of $\gamma$ ), given by $\epsilon_{\mathrm{j}}=(\sqrt{3} / 2) \mathrm{a}\left|\eta_{\mathrm{j}}\right|$.

The above result applies to all low-energy mini bands, except the first mini band of metallic CNTs, for which $\eta_{1}=0$. In this case, equation (8) yields

$$
\mathrm{v}_{\mathrm{x}}^{(\mathrm{n}, \mathrm{m})}(0, \mathrm{E})=\mathrm{v}_{0} \epsilon^{2}
$$

where

$$
\mathrm{v}_{0}=\frac{\sqrt{3} \mathrm{mn}\left(\left(\mathrm{m}^{2}-\mathrm{n}^{2}\right)(2 \mathrm{n}+\mathrm{m})(2 \mathrm{~m}+\mathrm{n})\right.}{4\left(\mathrm{n}^{2}+\mathrm{m}^{2}+\mathrm{mn}\right)^{3}} .
$$


This quadratic dependence on $\epsilon$ means that low-energy transverse currents in metallic CNTs is indeed small, in agreement with [4]. In contrast the square-root dependence arising when $\eta_{\mathrm{j}} \neq 0$ means that transverse currents in non-metallic CNTs are predicted to be much larger. This behaviour is illustrated in the exact results of Fig. 1. obtained by differentiating equation (1) with respect to the transverse and longitudinal wave vectors. For each mini band $\mathrm{q}$, the red curves of Fig. 1 show the dimensionless velocities $\mathrm{v}_{\mathrm{x}}\left(\mathrm{q}, \mathrm{k}_{\mathrm{y}}^{+}(\mathrm{q}, \mathrm{E})\right) / \mathrm{v}_{\mathrm{F}}$ as a function of $\mathrm{E}$ for the $(8, \mathrm{~m})$ family of CNTs. The black curves show the quantity $\mathrm{v}_{\mathrm{x}}^{(\mathrm{n}, \mathrm{m})}(\mathrm{E})$, obtained by adding the values of the red curves for each open channel of energy E. As expected, for the achiral $(8,8) \mathrm{CNT}_{\mathrm{x}}^{(\mathrm{n}, \mathrm{m})}(\mathrm{E})=0$, whereas for the chiral CNTs $v_{\mathrm{x}}^{(\mathrm{n}, \mathrm{m})}(\mathrm{E}) \neq 0$.
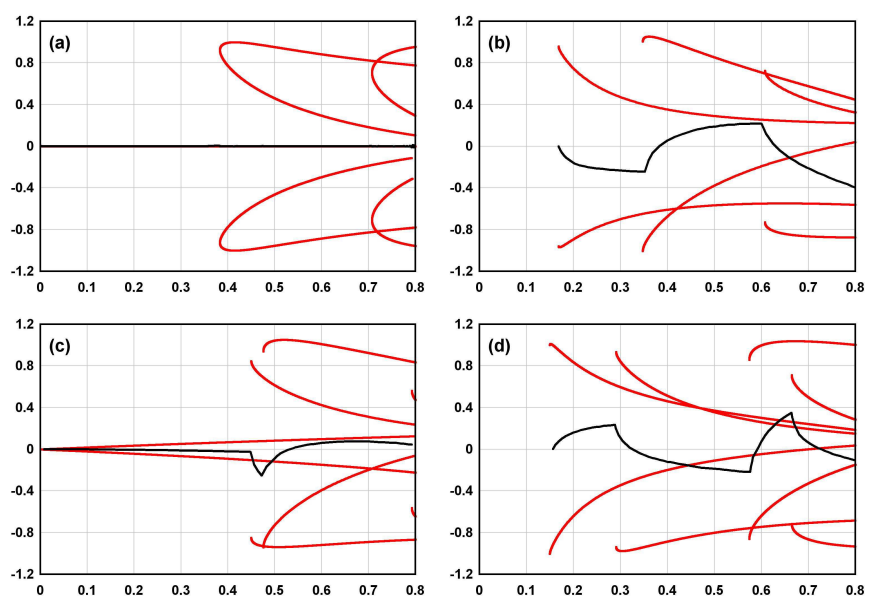

FIG. 1: Velocity components for the $(8, \mathrm{~m})$ family of CNTs plotted against the energy $\mathrm{E}(\mathrm{eV})$, where $\mathrm{m}=8,4,5,6$ in figs(a) to $(d)$ respectively. The red curves show the transverse velocities $\mathrm{v}_{\mathrm{x}}\left(\mathrm{q}, \mathrm{k}_{\mathrm{y}}^{+}(\mathrm{q}, \mathrm{E})\right) / \mathrm{v}_{\mathrm{F}}$ of right-moving electrons belonging to individual channels $q$. The black curves show the total velocity $\mathrm{v}_{\mathrm{x}}^{(\mathrm{n}, \mathrm{m})}(\mathrm{E})$ of Eq. (3).

As well as predicting the energy dependence of transverse velocities, equations (10) and (12) also yield the sign of $v_{x}^{(n, m)}\left(\eta_{j}, E\right)$. For example, when $n$ and $m$ are positive, the sign of $\mathrm{v}_{1}$ is equal to the sign of $(-\mathrm{X})$ and therefore for the first open mini band of a $(8,4) \mathrm{CNT}$, $\mathrm{v}_{\mathrm{x}}^{(8,4)}\left(\eta_{1}, \mathrm{E}\right)$ is negative, whereas for the first mini band of a $(8,6) \mathrm{CNT}, \mathrm{v}_{\mathrm{x}}^{(8,6)}\left(\eta_{1}, \mathrm{E}\right)$ is positive. Similarly, when $n \geq 0$ and $m \geq 0$, the sign of $v_{0}$ is equal to the sign of $(\mathrm{m}-\mathrm{n})$. Hence equation (12) shows that for the lowest mini band of a $(8,5) \mathrm{CNT}, \mathbf{v}_{\mathrm{x}}^{(10,4)}(0, \mathrm{E})<0$. For successive higher-energy mini bands, $\eta_{j}$ alternates in sign and therefore equations (9) and (10) reveal that the transverse velocities of successive higher-energy mini bands have a square-root $\epsilon$ dependence, with an alternating sign. In Eq. (3), the label q sums over $\mathrm{N}(\mathrm{E})$ right moving open channels, where $\mathrm{N}(\mathrm{E})$ is a discontinuous function of $\mathrm{E}$, which changes by an integer whenever new channels open or close. As predicted by equation (4), the red curves in Fig. 1, show that right-moving channels open or close in pairs and just as a pair of channels open, their tangential velocities cancel. Consequently, as shown in Fig. 1 $v_{x}^{(n, m)}(E)$ is a continuous function of $E$, with a discontinuous first derivative.

For the purpose of designing a CNT windmill with the largest torque, or a CNT with the largest internal magnetic field, it is of interest to compute the maximum integrated flux of transverse momentum carried by rightmoving electrons in an infinite chiral CNT. Since CNTs with $\eta_{1} \neq 0$ possess the most favourable energy dependence for $\mathrm{v}_{\mathrm{x}}^{(\mathrm{n}, \mathrm{m})}\left(\eta_{1}, \mathrm{E}\right)$, we focus on non-metallic CNTs. Since $\mathrm{v}_{\mathrm{x}}^{(\mathrm{n}, \mathrm{m})}\left(\eta_{1}, \mathrm{E}\right)$ and $\mathrm{v}_{\mathrm{x}}^{(\mathrm{n}, \mathrm{m})}\left(\eta_{2}, \mathrm{E}\right)$ have opposite signs, the sum $\mathrm{v}_{\mathrm{x}}^{(\mathrm{n}, \mathrm{m})}(\epsilon)=\mathrm{v}_{\mathrm{x}}^{(\mathrm{n}, \mathrm{m})}\left(\eta_{1}, \mathrm{E}\right)+\mathrm{v}_{\mathrm{x}}^{(\mathrm{n}, \mathrm{m})}\left(\eta_{2}, \mathrm{E}\right)$ increases monotonically with $\mathrm{E}$ for $\epsilon_{1} \leq \epsilon \leq \epsilon_{2}$ and then decreases with $\mathrm{E}$ for $\epsilon \geq \epsilon_{2}$, passing through zero when $\epsilon=\epsilon_{\max }$. Hence the maximum integrated flux of transverse velocity is proportional to

$$
\mathrm{v}_{\max }^{(\mathrm{n}, \mathrm{m})}=\int_{\epsilon_{1}}^{\epsilon_{\max }} \mathrm{d} \epsilon \mathrm{v}_{\mathrm{x}}^{(\mathrm{n}, \mathrm{m})}(\epsilon)
$$

From equation (91), one obtains $\epsilon_{\max }=15 \epsilon_{2} / 14$ and

$$
\mathrm{v}_{\max }^{(\mathrm{n}, \mathrm{m})}=\frac{\beta \mathrm{mn}(\mathrm{n}+\mathrm{m}) \cdot[\operatorname{sign} \text { of }(-\mathrm{X})]}{\left(\mathrm{n}^{2}+\mathrm{m}^{2}+\mathrm{mn}\right)^{5 / 2}}
$$

where $\beta=2 \sqrt{6} \pi^{2}\left[4 \arctan \left(\frac{1}{\sqrt{14}}\right)-\arctan \left(\sqrt{\frac{8}{7}}\right)\right] \approx$ 10.9 .

Equation (14) reveals that $\mathrm{v}_{\max }^{(\mathrm{n}, \mathrm{m})} \rightarrow 0$ as $\mathrm{n}, \mathrm{m} \rightarrow \infty$, which reflects the fact that the angular momentum carried by an electron wind is a consequence of the finite diameter of the CNT and the finite difference between successive values of $\eta_{\mathrm{j}}$. We also note that the optimum values of $(\mathrm{n}, \mathrm{m})$, which maximise $\mathrm{v}_{\max }^{(\mathrm{n}, \mathrm{m})}$ are those which possess a chiral angle close to $60^{\circ}\left(1-\frac{3}{\pi} \cos ^{-1} \sqrt{11 / 20}\right) \approx 18^{\circ}$.

Having analysed the transverse velocity of electrons in a chiral nanotubes, we now estimate the magnetic field generated by these electrons. In what follows we assume that the chiral CNT can be approximated by a long solenoid with a constant magnetic field $\mathrm{B}$ inside the CNT and zero field outside. Since the number of right-moving electrons per unit length in channel $\mathrm{q}$ is $(1 / \pi \hbar) \mathrm{dE} / \mathrm{v}_{\mathrm{y}}\left(\mathrm{q}, \mathrm{k}_{\mathrm{y}}^{+}(\mathrm{q}, \mathrm{E})\right)$, and these pass around the circumference of the CNT in a time $\tau=$ $|\mathbf{C h}| / \mathrm{v}_{\mathrm{x}}\left(\mathrm{q}, \mathrm{k}_{\mathrm{y}}^{+}(\mathrm{q}, \mathrm{E})\right)$, the contribution to the tangential current per unit length from the qth mini band is $\left[(1 / \pi \hbar) \mathrm{dE} / \mathrm{v}_{\mathrm{y}}\left(\mathrm{q}, \mathrm{k}_{\mathrm{y}}^{+}(\mathrm{q}, \mathrm{E})\right)\right] \mathrm{e} / \tau$. The magnetic field $\mathrm{B}$ inside a solenoid is $\mu_{0}$ multiplied by the tangential current per unit length. Hence the field due to all right-moving 
electrons in an energy window $\mathrm{eV}$ is

$$
\left.\mathrm{B}=\frac{2 \mathrm{e} \mu_{0}}{\mathrm{~h}|\mathbf{C h}|} \int_{0}^{\mathrm{eV}} \mathrm{dE} \sum_{\mathrm{q}}\left[\frac{\mathrm{v}_{\mathrm{x}}\left(\mathrm{q}, \mathrm{k}_{\mathrm{y}}^{+}(\mathrm{q}, \mathrm{E})\right)}{\mathrm{v}_{\mathrm{y}}\left(\mathrm{q}, \mathrm{k}_{\mathrm{y}}^{+}(\mathrm{q}, \mathrm{E})\right)}\right] \Theta\left(\mathrm{E}-\mathrm{E}_{\mathrm{q}}\right)\right),
$$

where $\mathrm{E}_{\mathrm{q}}$ is the lowest energy of the qth mini band. Since the current carried by these electrons is $\mathrm{I}=$ $\left.(1 / \pi \hbar) \int_{0}^{\mathrm{eV}} \mathrm{dE} \sum_{\mathrm{q}} \Theta\left(\mathrm{E}-\mathrm{E}_{\mathrm{q}}\right)\right)$, the magnetic field can be written $\mathrm{B}=\mu_{0} \mathrm{I} \alpha /|\mathbf{C h}|$, where

$$
\alpha=\frac{\left.\int_{0}^{\mathrm{eV}} \mathrm{dE} \sum_{\mathrm{q}}\left[\frac{\mathrm{v}_{\mathrm{x}}\left(\mathrm{q}, \mathrm{k}_{\mathrm{x}}^{+}(\mathrm{q}, \mathrm{E})\right)}{\mathrm{v}_{\mathrm{y}}\left(\mathrm{q}, \mathrm{k}_{\mathrm{y}}^{+}(\mathrm{q}, \mathrm{E})\right)}\right] \Theta\left(\mathrm{E}-\mathrm{E}_{\mathrm{q}}\right)\right)}{\left.\int_{0}^{\mathrm{eV}} \mathrm{dE} \sum_{\mathrm{q}} \Theta\left(\mathrm{E}-\mathrm{E}_{\mathrm{q}}\right)\right)} .
$$

The dimensionless parameter $\alpha$ is the average ratio of the tranverse and longitudinal group velocities. At low voltages, Eq. (4) allows this to be written as a sum over channels near the K-point $\mathbf{K}$ and using Eq. (9), one obtains

$$
\alpha=\frac{\int_{0}^{\mathrm{eV}} \mathrm{d} \epsilon \sum_{\mathrm{j}}\left[\frac{\mathrm{v}_{\mathrm{j}} \epsilon_{\mathrm{j}}}{\sqrt{2}}\right] \Theta\left(\epsilon-\epsilon_{\mathrm{j}}\right)}{\int_{0}^{\mathrm{eV}} \mathrm{d} \epsilon \sum_{\mathrm{j}} \Theta\left(\epsilon-\epsilon_{\mathrm{j}}\right)} .
$$

For $\epsilon_{1}<\mathrm{eV} / \gamma<\epsilon_{2}$, this yields $\alpha=\mathrm{v}_{1} \epsilon_{1} / \sqrt{2}$, which is of order unity for $(8,4)$ or $(8,6)$ CNTs. Consequently, for a current of $10^{-4}$ amps, $|\mathrm{B}| \approx 10^{-1}$ Teslas, which is large enough to produce significant spintronic effects [35], such as rotating the magnetic moment of a small magnetic island [36] or a metallocene encapsulated within a CNT [37]. By computing the energy stored in this magnetic field, we find an associated inductance per unit length of $L=\mu_{0} \alpha^{2} / 4 \pi$. This internal field could be detected through NMR measurements on encapsulated spins and could form the basis of scanning MR probe with nanometre spatial resolution. Finally we note that if the longitudinal current is driven by an ac voltage, the oscillations present in $\mathrm{v}_{\mathrm{x}}^{(\mathrm{n}, \mathrm{m})}(\mathrm{E})$ will lead to the generation of higher harmonics, which may provide an alternative probe of chiral currents.

[1] V. Kristic, G. Wagniere and G.L.J.A. Rikken, Chem. Phys. Lett. 39025 (2004)

[2] Y. Miyamoto, S.G. Louie, A. Rubio and M.L. Cohen, Phys. Rev. B 6013885 (1999)

[3] Z. C. Tu and X. Hu. Phys. Rev. B 72033404 (2005).

[4] Y. Miyamoto, S.G. Louie, and M.L. Cohen, Phys. Rev. Lett. 762121 (1996)

[5] Y. Myamoto, Phys. Rev. B54 16 (1996)

[6] O.M. Yevtushenko, G.Y S and S. Maksimenko, A. Lakhtakia and D. A. Romanov, Phys. Rev. Lett. 791102 (1997)

[7] P. Kral, E.L. Mele and D. Tomanek, Phys. Rev. Lett., 851512 (2000)

[8] P.C.P Watts and W.K. Hsu, Appl. Phys. A 7879 (2004)

[9] R.T. Senger, S. Dag and S. Ciraci, Phys. Rev. Lett. 93 196807 (2004)
[10] J. Cummings and A. Zettl, Science 289602 (2000).

[11] M-F Yu, B.I. Yakobson and R.S. Ruoff, J. Chem. B 104, 8764 (2000)

[12] J. W. Kang, K, O, Song, O, K, Kwon and H. J. Hwang. Nanotechnology 16, 2670, (2005).

[13] A. N. Kolmogorov and V. H. Crespi, Phys. Rev. Lett, 85, 4727, (2000).

[14] O. Lourie, D. M. Cox and H. D. Wagner. Phys. Rev. Lett. 81, 1638 (1998).

[15] L. Forro, Science 289560 (2000).

[16] P. G. Collins, M. S. Arnold and Ph. Avouris. Science 292, 706 (2001).

[17] B. Bourlon, D.C. Glattli, C. Miko, L. Forro and A. Bachtold, Nano Lett. 4, 709 (2004).

[18] A. Subramanian, L. X. Dong, J. Tharian, U. Sennhauser and B. J. Nelson. Nanotechnology 18075703 (2007).

[19] A.M. Fennimore, T. D. Yuzvinsky, W. Q. Han, M. S. Fuhrer, J. Cummings and A. Zettl, Nature 424408 (2003).

[20] S. B. Legoas, V. R. Coluci, S. F. Braga, P. Z. Coura, S. O. Dantes and D. S. Galvão. Phys.Rev. Lett, 90, 055504 (2003).

[21] Q. Zheng and Q. Jiang, Phys.Rev. Lett, 88, 045503 (2002)

[22] P.A. Williams, S. J. Papadakis, A. M. Patel, M. R. Falvo, S. Washburn and R. Superfine. Phys. Rev. Lett. 89255502 (2002).

[23] Y. Zhao, C. C. Ma, G. Chen and Q. Jiang. Phys. Rev. Lett. 91, 175504 (2003).

[24] V. V. Deshpande, H. Y. Chiu, H. W. Ch. Postma, C. Mikó, L. Forró and M. Brockrath. Nano Lett. 61092 (2006).

[25] I. M. Grace, S. W. Bailey and C. J. Lambert, Phys. Rev. B 70, 153405 (2004).

[26] R. Saito, R. Matsuo, T. Kimura, G. Dresselhaus and M. S. Dresselhaus. Cem. Phys. Lett. 348187 (2001).

[27] Yu. E. Lovovik, A. V. Minigin and A. M. Popov. Phys. Lett. A 313112 (2003).

[28] P. A. E. Schoen, J. H. Walther, S. Arcidiacono, D. Poulikakos and P. Koumoutsakos. Nano Lett. 6, 1910, (2006).

[29] A. Barriero, R. Rurali, E. R. Hérnandez, J. Moser, T. Pichler, L. Forró and A. Bachtold. Sciencexpress, Science 10.1126 (115559) (2008).

[30] P. Kral and H. R. Sadeghpour, Ohys. Rev. B. 65, 161401, (2002).

[31] S. W. D. Bailey, I. Amanatidis and C. J. Lambert. Phys. Rev. Lett. 100, 256802 (2008).

[32] K. Tsubaki, Y. Nakajima, T. Hanajiri, H. Yamaguchi, Journal of Physics: Conference Series 38 49(2006)

[33] R. Saito, G. Dresselhaus, M. S. Dresselhaus, Physical Properties of Carbon Nanotubes. Imperial College London, 1998.

[34] A. Kis, K. Jensen, S. Aloni, W. Mickelson and A. Zettl. Phys. Rev. Lett. 97, 025501 (2006).

[35] The corresponding magnetic flux $\phi=B|\mathbf{C h}|^{2} /(4 \pi)$ is given by $\phi / \phi_{0}=1.44 \sqrt{3} \times\left(n^{2}+m^{2}+n m\right)^{1 / 2} I \alpha$. For a current of $10^{-4}$ amps, $\phi / \phi_{0} \approx 10^{-4} \alpha$, which is too small to produce significant orbital effects.

[36] M. Hortamani, P. Kratzer and M. Scheffler. Phys. Rev. B 76235426 (2007).

[37] V. M. García-Suárez, J. Ferrer and C.J. Lambert, Phys. Rev. Lett. 96106804 (2006) 